\title{
On Jordan Algebras
}

\author{
Zanyar Anwer Ameen \\ Deptartment of Mathematics, College of Science, University of Duhok, \\ Kurdistan-Region, IRAQ \\ zanyar@uod.ac
}

\begin{abstract}
Jordan algebras are the most natural type of algebras associated with finitely additive measures. Some consequences of this class of algebras are found. In particular, the questions of, what is the nature of the $\sigma$-algebra generated by the Jordan algebra? when the Jordan algebra includes the Borel $\sigma$-algebra? and when the Jordan (measure) algebra is complete? are answered.
\end{abstract}

Keywords: Jordan algebra, Jordan field, Jordan measure algebra, Measure algebra 2010 MSC No: 28A05; 28A60 and 28A75.

\section{Introduction}

The concept of Jordan measure was first introduced by Giuseppe Peano (1858-1932) [17] and Camille Jordan (18381922) [13, independently. This type of measure plays a crucial role in developing Measure Theory. It has extended the notions of length, area and volume. This measure is defined on bounded subsets of a Euclidean space $\mathbb{R}^{n}$ which has the property that a set in $\mathbb{R}^{n}$ is Jordan measurable iff its boundary has (Lebesgue) measure zero. Based on this many mathematicians, including 2, defined the Jordan measurability of a subset on arbitrary topological measure space as: A subset $A$ of a measure space $(X, \sigma(\tau), \mu)$ is Jordan measurable iff $\mu(\partial(A))=0$, where $\partial$ stands for the topological boundary of a set. For a topological space $X$, the family of all Jordan measurable subsets forms an algebra of sets. Such an algebra is called the Jordan algebra and denoted by $\mathcal{J}(X)$ or briefly $\mathcal{J}$, for more details see [2, Chapter 3]. In [3], Armstrong obtained many results on Jordan algebras (under the name of Jordan fields) based on the work of Maharam [15, where the underlying space is the Stone space of a Boolean algebra. He gave a good characterization on when the Jordan field forms a $\sigma$-algebra. In this note, we present a parallel result on Jordan algebras of a more general class of topological spaces. Furthermore, we find some properties under which the Jordan algebra $\mathcal{J}$ (resp. the $\sigma$-algebra generated by it, $\sigma(\mathcal{J})$ ) contains the Borel $\sigma$-algebra $\mathbb{B}$. We also study completeness of the Jordan measure algebra $\mathcal{J}_{\mu}$ (resp. the $\sigma$-algebra generated by it, $\sigma\left(\mathcal{J}_{\mu}\right)$ ).

Let $(X, \tau)$ be a given topological space and let $A \subseteq X$. The notations $(A)$ and $(A)$ are, respectively, the interior and the closure of $A$. A subset $A$ of $(X, \tau)$ is nowhere dense if $((A))=\emptyset$. A meager set is a countable union of nowhere dense sets. A set $A \subseteq(X, \tau)$ is regular closed if $A=((A))$. A subset $A$ of $(X, \tau)$ is called $G_{\delta}$ if it is a countable intersection of open subsets of $X$. A space $(X, \tau)$ that has a countable dense subset is called separable. A space $(X, \tau)$ is called a second countable space if it has a countable base. A space $(X, \tau)$ is completely regular, if for every closed set $F$ in $X$ and every point $x \in X$ with $x \notin F$, there exist a continuous function $f: X \rightarrow[0,1]$, such that $f(x)=0$ and $f(F)=1$.

Let $X$ be a set and let $\mathcal{F}$ be a nonempty family of subsets of $X$. The smallest $\sigma$-algebra containing all the sets in $\mathcal{F}$ is called the $\sigma$-algebra generated by $\mathcal{F}$ and is denoted by $\sigma(\mathcal{F})$. For $(X, \tau)$, the Borel $\sigma$-algebra $\mathbb{B}=\sigma(\tau)$ is 
the $\sigma$-algebra generated by open sets in $X$, and the Baire $\sigma$-algebra $\mathbb{B}_{0}$ is the $\sigma$-algebra generated by all compact $G_{\delta}$ subsets of $X$. A Boolean algebra is called complete if every subset of it has a supremum.

The Borel measure $\mu$ is a $\sigma$-additive measure defined on the Borel $\sigma$-algebra $\sigma(\tau)$ of a space $X$. Null sets are sets of measure zero.

A measure $\mu$ on a topological space $(X, \tau)$ is called

(i) trivial if $\mu(X)=0$, otherwise it is nontrivial;

(ii) finite if $\mu(X)<\infty$;

(iii) nonatomic if $\mu(\{x\})=0$, for each $\{x\} \in X$; and

(iv) strictly positive if $\mu(G)>0$, for each $G \in \tau$.

(v) complete if each subset of a null set is measurable.

(vi) regular on $X$ if for every $B \in \mathbb{B}$ we have that $\mu(B)=\inf \{\mu(G): B \subseteq G, G$ is open $\}=\sup \{\mu(F): F \subseteq$ $B, F$ is closed $\}$.

(vii) Radon if $\mu(B)=\sup \{\mu(F): F \subseteq B, F$ is compact $\}$, for every $B \in \mathbb{B}$ and $X$ is (compact) Hausdorff.

The support of $\mu$, denoted by $\operatorname{supp}(\mu)$, is the set of all points $x \in X$ for which every open set $U$ containing $x$ is of positive measure. $\mu$ is a strictly positive measure on $X$ if $\operatorname{supp}(\mu)=X$. $(X, \tau)$.

Throughout this note, by the term "measure" we mean a finite Borel measure on arbitrary topological space

\section{Jordan Algebras}

In general, neither Jordan algebra $\mathcal{J}$ nor the $\sigma$-algebra generated by it, $\sigma(\mathcal{J})$, include the Borel $\sigma$-algebra $\mathbb{B}$, see Example 1, but the latter does contain the Baire $\sigma$-algebra $\mathbb{B}_{0}$. Namely, for any topological space $X, \sigma(\mathcal{J})$ includes $\mathbb{B}_{0}$, [15, Theorem 1(5)]. For those topological spaces that have $\mathbb{B}=\mathbb{B}_{0}, \sigma(\mathcal{J})$ includes $\mathbb{B}$. This is the situation as in $\mathbb{R}^{n}$, see [12, Theorem 2]. In this section we find some properties under which $\mathcal{J}$ and $\sigma(\mathcal{J})$ contain $\mathbb{B}$.

Example 2.1. Let $X$ be the space of ordinal $\omega_{1}+1$ with the usual order topology. For every Borel set A, define a measure $\mu$ by

$$
\mu(A)=\left\{\begin{array}{lc}
1, & \text { if } \omega_{1} \in A \\
0, & \text { otherwise } .
\end{array}\right.
$$

Then, by the remark on page 171 in [15], $\mathcal{J}$ and $\sigma(\mathcal{J})$ is identical to $\mathbb{B}_{0}$. The singleton $\left\{\omega_{1}\right\}$ is Borel because it is closed. But it is not Jordan as $\mu\left(\partial\left(\left\{\omega_{1}\right\}\right)\right)=1$.

Definition 2.2. [4] A measure $\mu$ on some topological space $(X, \tau)$ is said to be residual if $\mu(N)=0$ for every nowhere dense subset $N$ of $X$.

Theorem 2.3. Let $\mu$ a Radon measure on a compact Hausdorff space $X$. Then $\mu$ is residual if and only if the Jordan algebra $\mathcal{J}$ contains the Borel $\sigma$-algebra $\mathbb{B}$.

Proof. Assume that $\mu$ is residual. Let $E$ be a Borel measurable subset $X$. Clearly $E \backslash \operatorname{Int}(E)$ is Borel and does not contain any open set. By outer regularity of $\mu$,

$$
\mu(E \backslash \operatorname{Int}(E))=\sup \{\mu(K): \operatorname{compact} K \subseteq E \backslash \operatorname{Int}(E)\} .
$$

But every compact subset $K \subseteq E \backslash \operatorname{Int}(E)$ is nowhere dense and so $\mu(K)=0$ by assumption. Therefore $\mu(E \backslash \operatorname{Int}(E))=$ 0 . Hence $\mu(E)=\mu(\operatorname{Int}(E))$. By the same way above, using inner regularity, one can obtain $\mu(E)=\mu(\operatorname{Cl}(E))$. Thus $\mu(\partial(E))=0$, which shows that $E$ is Jordan measurable.

The converse can be followed from [1, Theorem 3.2]. 
Lemma 2.4. [1, Theorem 2.10] No nontrivial nonatomic uniformly regular measure can be also residual.

From the above two results we conclude the following:

Theorem 2.5. If $\mu$ is nontrivial nonatomic uniformly regular Radon measure on a compact Hausdorff space $X$, then $\mathcal{J}$ is only an algebra of sets. In particular, $\mathcal{J}$ neither includes the Baire $\sigma$-algebra nor the Borel $\sigma$-algebra.

Definition 2.6. [8] Let $\mu$ be a Radon measure on a compact Hausdorff space $X$ and $\mu_{0}$ its restriction to the Baire algebra of $X$, and let $\hat{\mu}$ be the completion of $\mu_{0} . \mu$ is said to be completion regular if every Borel subset of $X$ is $\hat{\mu}$-measurable.

Remark 2.7. In [3, Propsition 2.3] Armstrong proved that for a regular measure $\mu$ on a compact Hausdorff space, the Jordan algebra includes the Borel $\sigma$-algebra if and only if $\mu$ is completion regular. But this is not the case, the product measure $\lambda$ on the product space $\{0,1\}^{\omega}$ is completion regular [10, 417J] and the Jordan algebra is a proper subalgebra of the Borel $\sigma$-algebra of $\lambda$, see [2, Theorem 4.3.6]. On the other hand, the other direction still holds, i.e. if the Jordan algebra contains the Borel $\sigma$-algebra, $\mu$ is completion regular.

Definition 2.8. [5] A measure $\mu$ on a space $X$ is said to be uniformly regular if there is a countable family $\mathcal{K}$ of compact $G_{\delta}$ subsets of $X$ such that for each open set $U \subseteq X$ and each $\epsilon>0$, there is $K \in \mathcal{K}$ with $K \subseteq U$ such that

$$
\mu(U \backslash K)<\epsilon
$$

Proposition 2.1. Let $\mu$ be a strictly positive uniformly regular measure on a space $X$. Then $X$ is separable.

Proof. Let $\mathcal{K}=\left\{K_{n}: n \in \mathbb{N}\right\}$ be a family of compact $G_{\delta}$ subsets of $X$ that makes $\mu$ uniformly regular. For every $K_{n}$ choose an element $x_{n} \in K_{n}$. Set $D=\left\{x_{n}: n \in \mathbb{N}\right\}$. Obviously, $D$ is countable. It remains to show that this set is dense in $X$. Suppose otherwise that $X \neq(D)$. So $X \backslash(D)$ is a nonempty open subset in $X$. By assumption, there is a nonempty compact $G_{\delta}$ set $K_{n}$ such that $x_{n} \in K_{n} \subseteq X \backslash(D)$, which is contraction. Hence $X=(D)$. This proves that $X$ is separable.

Lemma 2.9 (Folklore). Let $(X, \tau)$ be a second countable topological space with a countable base $\mathscr{B}$. The Borel $\sigma$-algebra $\sigma(\tau)$ is equal to the $\sigma$-algebra generated by $\mathscr{B}$.

Proof. Since each element in $\mathscr{B}$ is open, then $\mathscr{B} \subseteq \tau$ and so $\sigma(\mathscr{B}) \subseteq \sigma(\tau)$. For other direction, let $U$ be an open subset of $X$. Then $U$ can be written as a union of countably many elements $B_{i} \in \mathscr{B}$. That is

$$
U=\bigcup_{i=1}^{\infty} B_{i} \quad \text { for each } B_{i} \in \mathscr{B} .
$$

Therefore $U \in \sigma(\mathscr{B})$. Hence $\sigma(\tau) \subseteq \sigma(\mathscr{B})$ and so $\sigma(\mathscr{B})=\sigma(\tau)$

Lemma 2.10. Let $(X, \tau)$ be a second countable topological space and let $\mathscr{B}$ be any its basis. Then $\sigma(\tau)=\sigma(\mathscr{B})$.

Proof. The first inclusion is immediate. That is $\sigma(\mathscr{B}) \subseteq \sigma(\tau)$ as $\mathscr{B} \subseteq \tau$. For other direction, since $X$ is second countable, the base $\mathscr{B}$ contains a countable base $\mathscr{B}_{0}$, say. By Lemma 1 , $\sigma(\tau)=\sigma(\mathscr{B} 0) \subseteq \sigma(\mathscr{B})$. Thus $\sigma(\mathscr{B})=$ $\sigma(\tau)$.

Proposition 2.2. Let $\mu$ be a strictly positive uniformly regular measure on a metrizable space $X$. Then $\sigma(\mathscr{B})=\sigma(\tau)$, where $\mathscr{B}$ is any basis for the topology $\tau$ on $X$.

Proof. According to Proposition 1.1, $X$ is separable. By [18, Theorem 16.11], separability implies second countability for metrizable spaces. Hence the result by Lemma 1 .

Lemma 2.11. [6, Proposition 8.2.8] Let $\mu$ be a measure on a completely regular space X. A family of open sets in Jordan algebra forms a base for the topology on $X$.

From Lemma 1 and Proposition 1.2 , we obtain

Theorem 2.12. Let $\mu$ be a strictly positive uniformly regular measure on a metrizable space $X$. Then the $\sigma$-algebra generated by Jordan algebra contains the Borel $\sigma$-algebra, i.e. $\mathbb{B} \subseteq \sigma(\mathcal{J})$. 
Remark 2.13. Note that in the above result, we have the problem with subsets of null sets, they are always Jordan measurable (with respect to the given measure) but not Borel. So if we assume the measure to be complete, we will have $\mathcal{J} \subseteq \mathbb{B}$ and hence $\sigma(\mathcal{J}) \subseteq \sigma(\mathbb{B})=\mathbb{B}$.

From this remark, we have the following corollary:

Corollary 2.14. Let $\mu$ be a strictly positive uniformly regular complete measure on a metrizable space $X$. Then the $\sigma$-algebra generated by Jordan algebra is exactly the Borel $\sigma$-algebra, i.e. $\mathbb{B}=\sigma(\mathcal{J})$.

Definition 2.15. [16] A measure $\mu$ on a compact Hausdorff space $X$ is said to be countably determined if there is a countable family $\mathcal{K}$ of compact subsets of $X$ such that for each open set $U \subseteq X$ and each $\epsilon>0$, there is $K \in \mathcal{K}$ with $K \subseteq U$ such that

$$
\mu(U \backslash K)<\epsilon
$$

Proposition 2.3. Let $\mu$ be a strictly positive countably determined complete measure on a compact Hausdorff space $X$. Then the $\sigma$-algebra generated by the family $\mathcal{K}$ is exactly the Borel $\sigma$-algebra, where $\mathcal{K}$ is a family of compact sets that makes $\mu$ uniformly regular on $X$.

Proof. Observe that a family $\mathcal{K}$ of compact sets that makes $\mu$ uniformly regular is countable. Then $\mathcal{K} \subseteq \mathbb{B}$, thus $\sigma(\mathcal{K}) \subseteq \mathbb{B}$. To prove the reverse of inclusion, let $U$ be an open set in $X$. By assumption, for each $\epsilon>0$, there is a compact set $K \in \mathcal{K}$ such that $K \subseteq U$ and $\mu(U \backslash K)<0$. Therefore,

$$
U=\bigcup_{K \subseteq U} K .
$$

Note that if for any case, there exists $x \in U$ but $x \notin K, \forall K \in \mathcal{K}$, we can add a compact set $\{x\}$ to some $K \in \mathcal{K}$ and refine our collection. Therefore $U \in \sigma(\mathcal{K})$, so $\mathbb{B} \subseteq \sigma(\mathcal{K})$. Hence $\mathbb{B}=\sigma(\mathcal{K})$. We are done.

Since, easily, one can show that every uniformly regular measure is countably determined, so the following result can be obtained:

Proposition 2.4. Let $\mu$ be a strictly positive uniformly regular complete measure on a compact Hausdorff space $X$. Then the $\sigma$-algebra generated by the family $\mathcal{K}$ is equal to the Borel $\sigma$-algebra, where $\mathcal{K}$ is a family of compact $G_{\delta}$ sets that makes $\mu$ uniformly regular on $X$.

Theorem 2.16. Let $\mu$ be a strictly positive uniformly regular complete measure on a compact Hausdorff space $X$. Then the $\sigma$-algebra generated by Jordan algebra is exactly the Borel $\sigma$-algebra. That is $\mathbb{B}=\sigma(\mathcal{J})$.

Proof. Let $\mathcal{K}$ be a countably family of compact $G_{\delta}$ sets that makes $\mu$ uniformly regular. By [2, Proposition 4.3 .2 (2)], the algebra $\mathcal{A}$ generated by $\mathcal{K}$ is a generator for $\mathcal{J}$. That is $\mathcal{K} \subset \mathcal{A} \subset \mathcal{J}$. Therefore, by Proposition 1.4 . $\sigma(\mathcal{K})=\mathbb{B} \subset \sigma(\mathcal{J})$, and the other inclusion follows from Remark 1 . Hence, $\sigma(\mathcal{J})=\mathbb{B}$.

Lemma 2.17. [9] For a point $x$ in a space $X$, then the Dirac measure $\delta_{x}$ is uniformly regular if and only if $x$ is $G_{\delta}$, the intersection of countably many open sets.

In Theorem 1, uniform regularity and completeness of measure cannot be removed, as shown in the following examples:

Example 2.18. Consider the completion of the measure $\mu$ given in Example 1 . Then $\mu$ can be seen as the Dirac measure for the point $\omega_{1}$ on the Borel $\sigma$-algebra of subsets of $X$. To prove that $\mu$ is not uniformly regular, by Lemma 1, it is enough to show that $\left\{\omega_{1}\right\}$ is not $G_{\delta}$. Let $\left\{\left[a_{n}, \omega_{1}\right]: n<\omega\right\}$ be a countable collection of (basic) open sets in $X$ containing $\omega_{1}$. Let $a>a_{n}$ for all $n<\omega$. This implies that $\left[a, \omega_{1}\right] \subseteq \bigcap_{n<\omega}\left[a_{n}, \omega_{1}\right]$ and so $\bigcap_{n<\omega}\left[a_{n}, \omega_{1}\right] \neq\left\{\omega_{1}\right\}$. Hence $\left\{\omega_{1}\right\}$ is not $G_{\delta}$, and $\sigma(\mathcal{J})=\mathbb{B}_{0} \neq \mathbb{B}$.

Example 2.19. The Borel measure $\mu$ on the unit interval $[0,1]$ is uniformly regular but not complete, and also $\sigma(\mathcal{J}) \neq \mathbb{B}$. The Cantor set $C \subset[0,1]$ is Jordan measurable and its subsets are also Jordan measurable. But many of these subsets are not Borel, see [7, Theorem C1]. 


\section{Jordan Measure Algebras}

Definition 3.1. The Jordan measure algebra of a measure space $(X, \mathbb{B}, \mu)$ is the Jordan algebra modulo null sets (sets of Jordan measure zero) and is denoted by $\mathcal{J}_{\mu}(X)$ or simply $\mathcal{J}_{\mu}$.

It is known that elements of $\mathcal{J}_{\mu}(X)$ are measurable classes, for more details, see [2, Section 3, Chapter 3].

Definition 3.2. The $\sigma$-algebra generated by Jordan measure algebra is defined by

$$
\sigma\left(\mathcal{J}_{\mu}(X)\right):=\left\{\bigvee_{n<\omega}\left[A_{n}\right]=\left[\bigcup_{n<\omega} A_{n}\right]: A_{n} \in \mathcal{J}(X)\right\}
$$

Notice that, in general, $\mathcal{J}_{\mu}$ (and even $\sigma\left(\mathcal{J}_{\mu}\right)$ ) is not complete as a Boolean algebra, see [2, Lemma 3.3.9(2)].

Theorem 3.3. Let $\mu$ be a Radon measure on a compact Hausdorff space $X$. Then $\mu$ is residual on $X$ if and only if $\mathcal{J}_{\mu}$ is complete.

Proof. Let $\mu$ be residual. By Theorem 1, $\mathcal{J}$ contains the Borel $\sigma$-algebra $\mathbb{B}$, which implies that $\mathcal{J}$ is a $\sigma$-algebra. By [11, Note 3, Page 169], $\mathcal{J}_{\mu}$ (forms a measure algebra) is complete.

Conversely, assume that $\mathcal{J}_{\mu}$ is complete. We need to show that $\mu$ is residual. Suppose otherwise that $\mu$ is not residual, without loss of generality, there is a closed nowhere dense set $N$ such that $\mu(N)>0$. It is known that every closed nowhere dense is boundary of an open set. There is an open subset $U$ of $X$ whose boundary is $N$. So $U$ is not Jordan measurable. On the other hand, by Lemma 1, $U=\bigcup_{\alpha} B_{\alpha}$, where each $B_{\alpha}$ is open Jordan measurable subsets of $X$. Thus $\mathcal{J}_{\mu}$ is not complete.

Lemma 3.4. [2, Theorem 4.3.6] Let $\mu$ be a strictly positive nonatomic uniformly regular Radon measure on a compact Hausdorff space $X$. Then the Jordan measure algebra of $\mu$ is isomorphic to the Jordan measure algebra of $\lambda$, the Lebesgue measure on $[0,1]$. That is

$$
\mathcal{J}_{\mu} \cong \mathcal{J}_{\lambda}
$$

Theorem 3.5. Let $\mu$ be a strictly positive uniformly regular nonatomic Radon measure on a compact Hausdorff space $X$. Then $\sigma\left(\mathcal{J}_{\mu}\right)$ is complete.

Proof. By Lemma 2, $\mathcal{J}_{\mu}$ is isomorphic to the Jordan measure algebra $\mathcal{J}_{\lambda}$. By [2, Lemma 3.3.8 (2)], $\sigma\left(\mathcal{J}_{\lambda}\right)$ is the Lebesgue measure algebra and by [14, Proposition 3.8] it is complete. Hence $\sigma\left(\mathcal{J}_{\mu}\right)$ is complete.

\section{Conclusion}

Discussing the Jordan algebra $\mathcal{J}$ and the $\sigma$-algebra generated by it, $\sigma(\mathcal{J})$, we have seen that generally none of these algebras contain the Borel $\sigma$-algebra $\mathbb{B}$. We have shown if a measure $\mu$ on a compact Hausdorff space $X$ is residual Rodan (resp. complete uniformly regular) that $\mathcal{J}$ (resp. $\sigma(\mathcal{J})$ ) contain $\mathbb{B}$. Moreover, we have recalled that, in general, both Jordan measure algebra $\mathcal{J}_{\mu}$ and the $\sigma$-algebra generated by it, $\sigma\left(\mathcal{J}_{\mu}\right)$, are not complete as Boolean algebras. We have proved that if a measure $\mu$ on a compact Hausdorff space $X$ is residual Rodan (resp. nonatomic uniformly regular), then $\mathcal{J}_{\mu}$ (resp. $\left.\sigma\left(\mathcal{J}_{\mu}\right)\right)$ is complete.

ACKNOWLEDGEMENTS. The author is grateful to the referee for his sincere evaluation and constructive comments which considerably improved the quality of the paper.

\section{References}

[1] Z. A. Ameen. Some results on the existence of residual measures. Journal of University of Duhok, 21(1):111-114.

[2] Z. A. Ameen. Finitely Additive Measures on Topological Spaces and Boolean Algebras. PhD thesis, University of East Anglia, 2015.

[3] T. E. Armstrong. Notes on Jordan fields based on an article by Maharam. Journal of Mathematical Analysis and Applications, 154(1):184-202, 1991. 
[4] T. E. Armstrong, K. Prikry, et al. Residual measures. Illinois Journal of Mathematics, 22(1):64-78, 1978.

[5] A. Babiker. Uniform regularity of measures on compact spaces. Journal fr Mathematik. Band, (289):188-198, 1977.

[6] V. I. Bogachev. Measure Theory, volume 2. Springer, 2007.

[7] F. Burk. Lebesgue measure and integration: an introduction, volume 32. John Wiley \& Sons, 2011.

[8] J. Choksi and D. Fremlin. Completion regular measures on product spaces. Mathematische Annalen, 241(2):113128, 1979.

[9] M. Dzamonja and G. Plebanek. On Efimov spaces and Radon measures. Topology and its Applications, 154(10):2063-2072, 2007.

[10] D. H. Fremlin. Measure theory, volume 4. Torres Fremlin, 2000.

[11] P. R. Halmos. Measure theory, volume 2. van Nostrand New York, 1950.

[12] K. Johnson. The $\sigma$-algebra generated by the Jordan sets in $\mathbb{R}^{n}$. Real Analysis Exchange, pages 278-282, 1993.

[13] C. Jordan. Remarques sur les intégrales définies. Journal de mathématiques pures et appliquées, 8:69-100, 1892.

[14] T. Lando. First order s4 and its measure-theoretic semantics. Annals of Pure and Applied Logic, 166(2):187-218, 2015.

[15] D. Maharam. Jordan fields and improper integrals. Journal of mathematical analysis and applications, 133(1):163-194, 1988.

[16] S. Mercourakis. Some remarks on countably determined measures and uniform distribution of sequences. Monatshefte für Mathematik, 121(1-2):79-111, 1996.

[17] G. Peano. Applicazioni geometriche del calcolo infinitesimale, volume 2. Fratelli Bocca, 1887.

[18] W. Stephen. General topology. Addison-Wesley, 1970. 